\title{
Let-7a microRNA functions as a potential tumor suppressor in human laryngeal cancer
}

\author{
XIAO-BO LONG ${ }^{1 *}$, GUANG-BIN SUN ${ }^{2 *}$, SHUANG HU ${ }^{1}$, GENG-TIAN LIANG ${ }^{2}$, NAN WANG ${ }^{1}$, \\ XIN-HAO ZHANG ${ }^{1}$, PING-PING CAO ${ }^{1}$, HONG-TAO ZHEN $^{1}$, YONG-HUA CUI ${ }^{1}$ and ZHENG LIU ${ }^{1}$ \\ ${ }^{1}$ Department of Otolaryngology-Head and Neck Surgery, Tongji Hospital, Tongji Medical College, \\ Huazhong University of Science and Technology, Wuhan; ${ }^{2}$ Department of Otolaryngology, \\ Gongli Hospital Pudong New Area, Shanghai, P.R. China
}

Received May 6, 2009; Accepted July 1, 2009

DOI: 10.3892/or_00000554

\begin{abstract}
MicroRNAs (miRNAs) are endogenously expressed non-coding RNAs, which are involved in the gene expression regulation. Lethal-7a (let-7a) is a founding member of miRNA family and recently it was found to be associated with several cancers, such as lung and colon cancers. In the present study, we found that let-7a miRNA expression was significantly downregulated both in human laryngeal squamous cancer tissues and in Hep-2 cells, a laryngeal cancer cell line, as compared with adjacent normal tissues and BEAS-2B cells, respectively. Moreover, we found that let-7a expression levels were significantly further decreased in non-differentiated $\left(\mathrm{G}_{3}\right)$ cancer tissues as compared with moderately and well differentiated cancer tissues $\left(G_{2}\right.$ and $\left.G_{1}\right)$, although no significant difference in let-7a expression levels between the cancer specimens with different $\mathrm{T}$ stages or specimens from patients with different lymph node metastasis status was revealed. In Hep- 2 cells, let-7a mimics transfection markedly suppressed proliferation and induced apoptosis of Hep-2 cells under the treatment of diamminedichloroplatinum or not and downregulated RAS and c-MYC protein expression without affecting the mRNA levels. In parallel, RAS and c-MYC protein levels were found significantly upregulated only in cancer tissues with downregulated let-7a expression. Thus, we
\end{abstract}

Correspondence to: Dr Zheng Liu or Dr Yong-Hua Cui, Department of Otolaryngology- Head and Neck Surgery, Tongji Hospital, Tongji Medical College, Huazhong University of Science and Technology, 1095 Jiefang Avenue, Wuhan 430030, P.R. China E-mail: zhengliuent@hotmail.com

E-mail: yhcui@tjh.tjmu.edu.cn

*Contributed equally

Abbreviations: miRNA, microRNA; let-7, lethal-7; DDP, diamminedichloroplatinum; MTT, methylthiazolyldiphenyltetrazolium

Key words: microRNA, lethal-7a, laryngeal cancer, proliferation, apoptosis, RAS, c-MYC propose that let-7a may be a tumor suppressor in laryngeal cancer by inhibiting cell growth, inducing cell apoptosis and downregulating the oncogenes expression.

\section{Introduction}

MicroRNAs (miRNAs) are a recently discovered group of small non-coding RNA molecules involved in the gene expression regulation. They are 19-25 nucleotides in length and control the expression of a wide variety of genes by binding to the complementary sequences in the 3'-untranslated regions of the target mRNAs, which results in a reduced translation rate and/or increased degradation of the mRNA (1). An involvement of miRNAs has been documented for almost all major cellular functions such as cell proliferation, cell differentiation, stress response, apoptosis and transcriptional regulation (2-4). Since corruption of these biological processes is the hallmark of many cancers, dysregulation of miRNA biogenesis and function may lead to tumorigenesis. In fact, recently, some miRNAs levels have been reported to be altered in certain cancers and some miRNAs have been revealed to be mapped to cancer-related genomic regions in chromosomes. The identification of several miRNAs as oncogenes or tumor suppressors underscores the potential significance of miRNAs in therapeutic and diagnostic applications $(5,6)$.

Lethal-7 (let-7), a founding member of miRNA family, is required for timing of cell fate determination in $C$. elegans $(7,8)$. In humans, similar to in C. elegans, the expression of let-7 is barely detectable in embryonic tissues but increases in differentiated and mature tissues (9). Recent studies demonstrated that expression levels of let-7 miRNA were reduced in human lung and colon cancers $(10,11)$ and let-7 might act as an anti-cancer molecule as it was able to repress RAS and/or c-MYC expression at the translational level $(3,12)$. Laryngeal carcinoma is a common malignant tumor in head and neck. Although its pathogenesis remains largely unknown; inactivation of tumor suppressors and amplification of oncogenes, including RAS and c-MYC, which regulate various intracellular signaling pathways, have been linked with its development (13-15). However, whether miRNAs, especially let-7, play a role in the pathogenesis of malignant tumors in head and neck including laryngeal carcinoma has not yet been 
investigated. The let-7 gene family consists of 11 very closely related genes and let-7a is currently the best characterized member. Let-7a is abundant in differentiated adult tissues and inappropriate expression of let-7a may result in oncogenic loss of differentiation $(8,16)$. In the present study, we detected the let-7a levels in laryngeal cancer tissues and a laryngeal cancer cell line Hep-2 and investigated the direct effect of let-7a on proliferation and apoptosis of Hep- 2 cells. Furthermore, we explored the relationship between let-7a and RAS and c-MYC expression in laryngeal cancer tissues and Hep-2 cells.

\section{Materials and methods}

Tumor samples and patients. Forty-eight patients (42 male) who underwent surgery for primary laryngeal squamous carcinoma were recruited. The average age of patients at surgery was 58.2 years (range, $44-72$ years). None underwent chemotherapy or radiotherapy prior to surgery. The TNM stage of each patient was determined according to the classification of the International Union Against Cancer as follows: $\mathrm{T}_{1}, 8$ cases; $\mathrm{T}_{2}, 13$ cases; $\mathrm{T}_{3}, 18$ cases; and $\mathrm{T}_{4}, 9$ cases; and cervical lymph node metastasis was observed in 12 patients, including 10 cases in $\mathrm{N}_{1}$ stage and 2 cases in $\mathrm{N}_{2}$ stage. The grade of histopathological differentiation of tumor was determined according to World Health Organization criteria as follows: $G_{1}, 15$ cases; $G_{2}, 17$ cases; and $G_{3}, 16$ cases. The cancer tissues and corresponding adjacent normal tissues were collected during surgery. Each specimen was cut into 2 parts. One was immediately snap-frozen in liquid nitrogen and stored at $-70^{\circ} \mathrm{C}$ for subsequent miRNA study and the other one was fixed with $4 \%$ paraformaldehyde and embedded in paraffin for pathological and immunohistochemical study. All specimens were subjected to histological evaluation by a pathologist. All tumor tissues were confirmed to be squamous cell carcinoma and no cancer cell was found in all normal samples adjacent to the tumors.

All patients provided informed consent before their participation and this study was approved by the Ethics Committee of Tongji Medical College of Huazhong University of Science and Technology.

Cell culture and transfection. The human laryngeal cancer cell line Hep-2, derived from epidermoid carcinoma of the larynx and the human normal bronchial epithelial cell line BEAS-2B, derived from human bronchial epithelium transformed by an adenovirus 12-SV40 hybrid virus, were obtained from American Type Culture Collection (Manassas, VA, USA). Hep-2 cells were cultured in RPMI-1640 supplemented with $10 \%$ heat-inactivated fetal bovine serum (Gibco, Carlsbad, CA, USA) and BEAS-2B cells were cultured in DMEM/F12 (Gibco) supplemented with 5\% heat-inactivated fetal bovine serum. All cells were incubated at $37^{\circ} \mathrm{C}$ with $5 \%$ $\mathrm{CO}_{2}$ in humidified air. Hep- 2 cells were seeded in 6-well plates at a concentration of $2 \times 10^{5}$ cells per well. Twenty-four hours later, Hep-2 cells approaching 30-40\% confluence were transfected with let-7a mimics. The miRNA let-7a mimics belong to double-stranded small RNA products and were ready for use. The mature miRNA let-7a sequence is: UGA GGU AGU AGG UUG UAU AGU U (Ribobio, Guangzhou, China).
The let-7a mimics at a concentration of $50 \mathrm{nM}$ were used for the transfection through employing Lipofection 2000 (Invitrogen, Carlsbad, CA, USA) according to the manufacturer's protocol. Hep-2 cells transfected with non-specific control miRNA were served as controls. Forty-eight hours after transfection, cells were subjected to drug treatment experiments or collected for Western blot analysis and quantitative RT-PCR analysis.

MiRNA isolation and quantitative real-time PCR of let-7a. The miRNAs were isolated from tissues and cells using mirVana miRNA isolation kit (Ambion, Austin, TX, USA) following the manufacturer's protocol. In the reverse transcription step, cDNA was reverse transcribed using let-7aspecific miRNA primer (Applied Biosystems, Foster City, CA, USA) and TaqMan MicroRNA reverse transcription kit (Applied Biosystems). The RT reactions were performed at $16^{\circ} \mathrm{C}$ for $30 \mathrm{~min}, 42^{\circ} \mathrm{C}$ for $30 \mathrm{~min}$ and $85^{\circ} \mathrm{C}$ for $5 \mathrm{~min}$. The quantitative real-time PCR reactions using the TaqMan MicroRNA assay (Applied Biosystems) together with the TaqMan Universal PCR Master Mix (Applied Biosystems) were performed on Fast Real-time PCR 7700 System (Applied Biosystems) at $95^{\circ} \mathrm{C}$ for $10 \mathrm{~min}$, followed by 40 cycles of $95^{\circ} \mathrm{C}$ for $15 \mathrm{sec}$ and $60^{\circ} \mathrm{C}$ for $1 \mathrm{~min}$. The human U6 RNA (Applied Biosystems) was amplified in parallel as an internal control. Relative gene expression was calculated using the comparative CT method, as described previously (17).

Methylthiazolyldiphenyl-tetrazolium analysis of cell proliferation. Cell viability was assessed by methylthiazolyldiphenyl-tetrazolium (MTT) assay. Approximately $10^{4} \mathrm{Hep}-2$ cells without transfection and cells transfected with let-7a or control miRNA were seeded in 96-well plates for $24 \mathrm{~h}$ and then exposed to various concentrations of diamminedichloroplatinum (DDP) $(0,5,10,15$ and $20 \mu \mathrm{g} / \mathrm{ml}$, Sigma, St. Louis, MO) for $48 \mathrm{~h}$. The cells were then incubated in $0.1 \mathrm{mg} / \mathrm{ml}$ solution of MTT (Sigma) at $37^{\circ} \mathrm{C}$ for $4 \mathrm{~h}$ and lysed in $150 \mu 1$ dimethyl sulfoxide (Sigma) at room temperature for $30 \mathrm{~min}$. Spectrometric absorbance at a wavelength of $492 \mathrm{~nm}$ was measured on $\mu$ Quant $^{\mathrm{TM}}$ Microplate Spectrophotometer (BioTek, Winooski, VT, USA).

Annexin V binding assay. Cell apoptosis was assessed by using Annexin V-FITC and propidium iodide (PI) double stain detection kit (Keygentec, Nanjing, China). Approximately $10^{5}$ Hep-2 cells without transfection and cells transfected with let-7a or control miRNA were seeded in 12-well plates for $24 \mathrm{~h}$ and then exposed to DDP at a concentration of 0 or $10 \mu \mathrm{g} / \mathrm{ml}$. Thereafter, the cells were trypsinized, washed with icecold PBS and resuspended in $500 \mu 1$ binding buffer. Then cells were stained with $5 \mu \mathrm{l}$ Annexin V-FITC and $5 \mu 1$ PI for $15 \mathrm{~min}$ in the dark at room temperature. Fluorescence was measured by FASCort flow cytometer (Becton-Dickinson, NY, USA) and processed with Cell Quest software (BectonDickinson) for analysis. Ten thousand cells were counted for each sample.

Quantitative real-time PCR of RAS and $c-M Y C$. The total RNAs were extracted from Hep-2 cells by using TRIzol reagent (Invitrogen) and treated by using a DNA-free kit 
(Ambion) to remove contaminating DNA. cDNA was reverse transcribed from $0.5 \mu \mathrm{g}$ of total RNA with random hexamer primers (Takara Biotechnology, Dalian, China). Quantitative PCR was performed by using the SYBR Premix Ex Taq kit (Takara Biotechnology) with the specific primers on Fast real-time PCR 7700 System (Applied Biosystems). The PCR conditions were $95^{\circ} \mathrm{C}$ for $10 \mathrm{~min}$, followed by 40 cycles of $95^{\circ} \mathrm{C}$ for $15 \mathrm{sec}, 60^{\circ} \mathrm{C}$ for $30 \mathrm{sec}$ and $72^{\circ} \mathrm{C}$ for $30 \mathrm{sec}$. GAPDH was amplified in parallel as an internal control. The genespecific primers are as follows: for RAS, sense: 5'-TGG ATA GCA TGA ATT CTG CAT TGA G-3'; antisense: 5'-TTA TCC CAA ACA GGC ACT TCA AAC-3'; for c-MYC, sense: 5'-CGG ATT CTC TGC TCT CCT CGA C-3'; antisense: 5'-CCT CCA GCA GAA GGT GAT CCA-3'; and for GAPDH: sense: 5'-ACC CAG AAG ACT GTG GAT GG-3'; antisense: 5'-TTC TAG ACG GCA GGT CAG GT-3'. Relative gene expression was calculated using the comparative CT method, as described previously (17).

Western blotting of RAS and $c-M Y C$. The cells were homogenized in chilled lysis buffer and stood for $30 \mathrm{~min}$ on ice. After centrifugation at $14,000 \mathrm{rpm}$ for $20 \mathrm{~min}$ at $4^{\circ} \mathrm{C}$, the supernatants were collected as protein samples. Approximately $60 \mu \mathrm{g}$ of lysate protein were subjected to electrophoresis with a 10\% SDS-polyacrylamide gel and then electroblotted onto a nitrocellulose filter membrane. After blockage of non-specific binding sites, the membrane was incubated with rabbit antihuman RAS or c-MYC antibody (Boster, Wuhan, China) at a dilution of 1:400 overnight at $4^{\circ} \mathrm{C}$. The membranes were then washed 3 times, incubated further with alkaline phosphatase conjugated goat anti-rabbit antibody (Jackson, West Grove, PA, USA) at $37^{\circ} \mathrm{C}$ for $1 \mathrm{~h}$. The immunoblots were visualized by use of a 5-bromo-4-chloro-3-indolylphosphate/nitroblue tetrazolium alkaline phosphatase color development kit (Roche, Indianapolis, IN, USA).

Immunohistochemistry. RAS and c-MYC protein expression in laryngeal cancer and normal tissues was detected using the streptavidin-peroxidase complex method with a histostain-plus kit (Zhongshan Golden Bridge Biotechnology, Beijing, China) as described (17). Rabbit anti-human RAS and cMYC antibodies (Boster) at a dilution of 1:400 were used as primary antibodies. Color development was achieved with 3', 3'-diaminobenzidine, which rendered positive cells brown. Normal rabbit serum and secondary antibody alone were used as negative controls. The number of RAS and c-MYC-positive cells was determined by counting 10 randomly selected fields in a blinded fashion at $\mathrm{x} 400$ magnification and expressed as the number of positive cells per high power field.

Statistical analysis. Data are presented as mean \pm SD. All data were confirmed with normal distribution and assessed for significance by paired or unpaired t-test or ANOVA as appropriate. Differences were considered statistically significant at $\mathrm{P}$-value $<0.05$.

\section{Results}

Decreased expression of let-7a miRNA in human laryngeal cancer and Hep-2 cells. Mature let-7a miRNA expression in

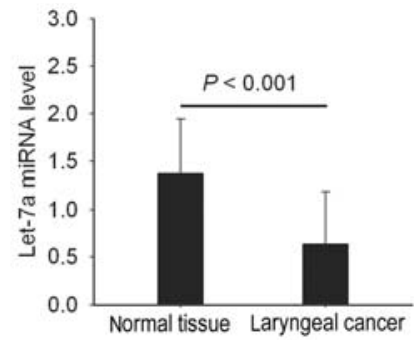

Figure 1. Decreased overall expression levels of let-7a in laryngeal cancer tissues compared with paired adjacent normal tissues determined by quantitative RT-PCR assay $(\mathrm{n}=48)$.

Table I. Relative expression levels of let-7a in laryngeal cancer patients with different $\mathrm{T}$ stages.

\begin{tabular}{lcc}
\hline & \multicolumn{2}{c}{ Relative let-7a expression levels } \\
\cline { 2 - 3 } T stage $(\mathrm{n}=48)$ & Adjacent normal tissue & Cancer tissue \\
\hline $\mathrm{T}_{1}(\mathrm{n}=8)$ & $1.54 \pm 0.55$ & $0.65 \pm 0.83^{\mathrm{a}}$ \\
$\mathrm{T}_{2}(\mathrm{n}=13)$ & $1.23 \pm 0.55$ & $0.58 \pm 0.46^{\mathrm{a}}$ \\
$\mathrm{T}_{3}(\mathrm{n}=18)$ & $1.45 \pm 0.60$ & $0.57 \pm 0.45^{\mathrm{a}}$ \\
$\mathrm{T}_{4}(\mathrm{n}=9)$ & $1.28 \pm 0.59$ & $0.58 \pm 0.52^{\mathrm{a}}$
\end{tabular}

${ }^{\mathrm{a}} \mathrm{P}<0.05$ compared with corresponding normal tissues in each group.

Table II. Relative expression levels of let-7a in laryngeal cancer patients with different node metastasis status.

Relative let-7a expression levels

Node status $(n=48)$ Adjacent normal tissue Cancer tissue

\begin{tabular}{lll}
\hline $\mathrm{N}=0(\mathrm{n}=36)$ & $1.34 \pm 0.59$ & $0.61 \pm 0.55^{\mathrm{a}}$ \\
$\mathrm{N}>0(\mathrm{n}=12)$ & $1.47 \pm 0.52$ & $0.54 \pm 0.47^{\mathrm{a}}$ \\
\hline
\end{tabular}

${ }^{\text {aP }}<0.05$ compared with corresponding normal tissues in each group.

the paired cancer and normal tissue samples from 48 patients with laryngeal squamous cell carcinoma were detected. Compared with adjacent normal tissues, the expression levels of let-7a miRNA in tumors were decreased in 37 of the 48 patients. Generally, the overall expression levels of let-7a were significantly downregulated at 2.16-fold in tumor tissues compared with adjacent normal tissues (Fig. 1). When dividing the tumors into different groups according to clinical stage and pathological grade, the let-7a levels were found to be markedly reduced in all tumors with different clinical stages and pathological grades when compared with corresponding normal tissues (Tables I, II and III). When comparing the difference in let-7a levels in cancer tissues between patients with different characteristics, no significant difference was found between patients with different $\mathrm{T}$ stages or lymph node metastasis statuses (Tables I and II); however, let-7a levels were significantly lower in non-differentiated $\left(\mathrm{G}_{3}\right)$ cancer 
Table III. Relative expression levels of let-7a in laryngeal cancer with different histopathological grades.

\begin{tabular}{lcc}
\hline & \multicolumn{2}{c}{ Relative let-7a expression levels } \\
\cline { 2 - 3 } Grade $(n=48)$ & Adjacent normal tissue & Cancer tissue \\
\hline $\mathrm{G}_{1}(\mathrm{n}=15)$ & $1.35 \pm 0.56$ & $0.77 \pm 0.48^{\mathrm{a}}$ \\
$\mathrm{G}_{2}(\mathrm{n}=17)$ & $1.62 \pm 0.60$ & $0.75 \pm 0.60^{\mathrm{a}}$ \\
$\mathrm{G}_{3}(\mathrm{n}=16)$ & $1.13 \pm 0.46$ & $0.25 \pm 0.31^{\mathrm{a}, \mathrm{b}, \mathrm{c}}$ \\
\hline
\end{tabular}

${ }^{a} \mathrm{P}<0.05$ compared with corresponding normal tissues in each group; ${ }^{b} \mathrm{P}<0.01$ compared with cancer tissues with $\mathrm{G}_{1}$ grade; ${ }^{\mathrm{c}} \mathrm{P}<0.01$ compared with cancer tissues with $\mathrm{G}_{2}$ grade.

tissues than in moderately and well differentiated cancer tissues $\left(\mathrm{G}_{2}\right.$ and $\left.\mathrm{G}_{1}\right)$ (Table III). As to the 11 patients without downregulated let-7a levels in cancer tissues compared with adjacent normal tissues, the TNM stage for those patients was as follows: $\mathrm{T}_{1} \mathrm{~N}_{0}, 1$ cases; $\mathrm{T}_{1} \mathrm{~N}_{1}, 1$ cases; $\mathrm{T}_{2} \mathrm{~N}_{0}, 3$ cases; $\mathrm{T}_{2} \mathrm{~N}_{1}$, 1 cases; $\mathrm{T}_{3} \mathrm{~N}_{0}, 2$ cases; $\mathrm{T}_{3} \mathrm{~N}_{1}, 2$ cases; and $\mathrm{T}_{4} \mathrm{~N}_{0}, 1$ cases. The

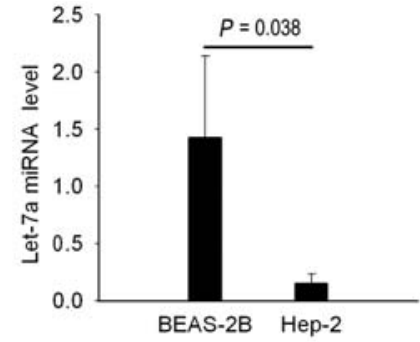

Figure 2. Decreased let-7a expression in Hep-2 cells compared with BEAS-2B cells detected by quantitative RT-PCR assay (three independent experiments).

histopathological differentiation grade of these samples was: $\mathrm{G}_{1}, 3$ cases; $\mathrm{G}_{2}, 4$ cases; and $\mathrm{G}_{3}, 3$ cases. No difference in proportions of patients with downregulated let-7a levels was found among patients with different TNM stage or histological grade (data not shown).

The baseline expression levels of let-7a miRNA in Hep-2 cells were determined in comparison with BEAS-2B cells. Consistent with findings in human tissues, significantly lower levels of let-7a (9.47-fold decrease) were found in Hep-2 cells than in BEAS-2B cells (Fig. 2).
A

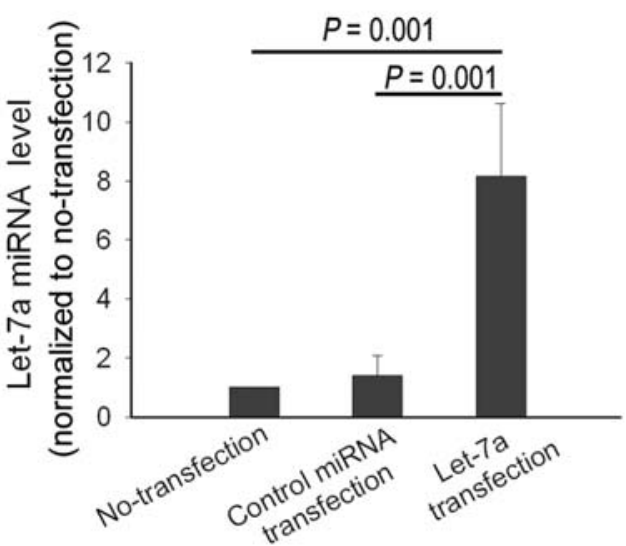

C

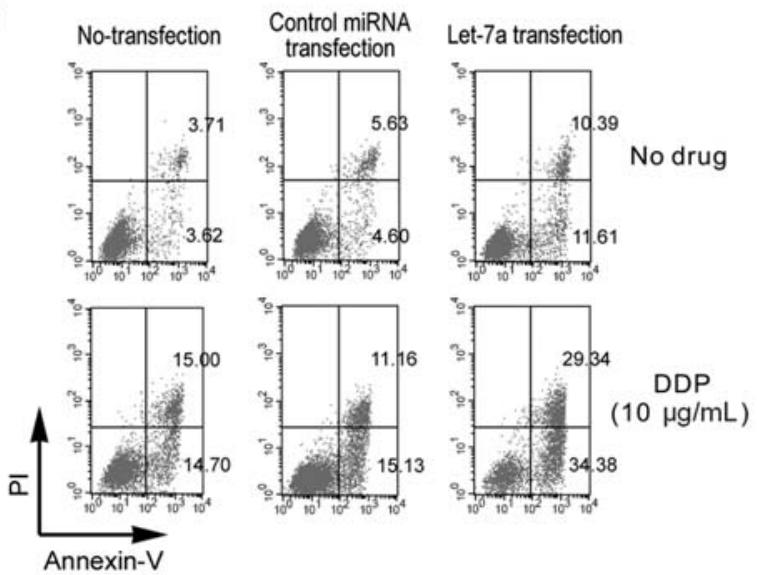

B
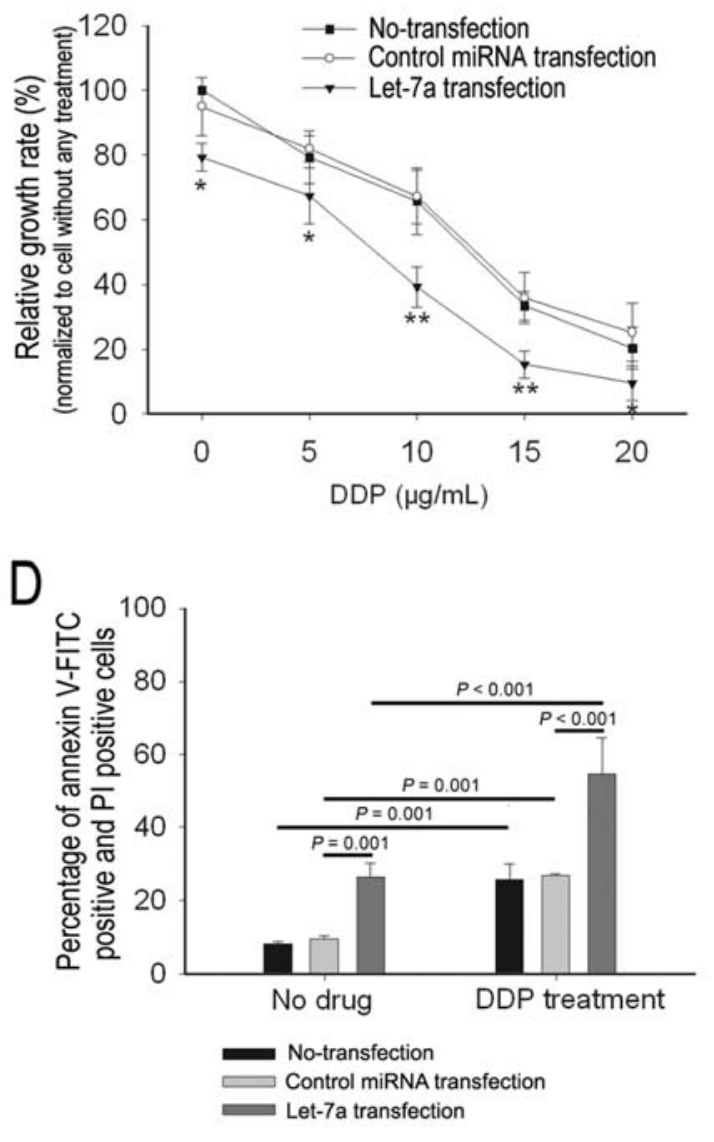

Figure 3. Let-7a miRNA inhibits cell proliferation and increase apoptosis of Hep-2 cells. (A) The let-7a expression $48 \mathrm{~h}$ after transfection with let-7a mimics in Hep-2 cells (three independent experiments). (B) The cells transfected with let-7a mimics or control miRNA were exposed to various concentrations of diamminedichloroplatinum (DDP) for $48 \mathrm{~h}$. Cell survival was then assessed by methylthiazolyldiphenyl-tetrazolium method normalized to samples without transfection and DDP treatment (three independent experiments). ${ }^{*} \mathrm{P}<0.05$ and ${ }^{* *} \mathrm{P}<0.01$ compared with control miRNA-transfected Hep-2 cells at the same DDP concentration. (C) Let-7a increases the apoptotic cell death in Hep-2 cells. The cells transfected with let-7a mimics or control miRNA were treated with DDP or not for $48 \mathrm{~h}$ followed by Annexin V binding assay. The number represents the percentage of cells in the quadrant. One representative experiment of the three independent experiments is shown. (D) Statistical analysis of Annexin V and PI-positive cells in different groups (three independent experiments). 
A

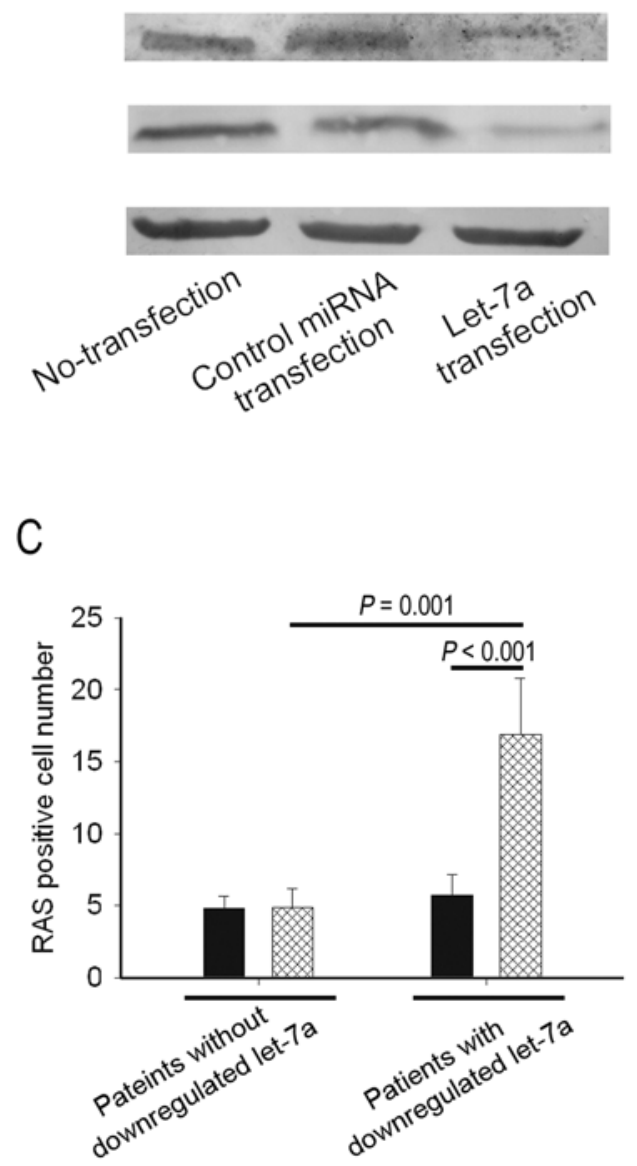

B

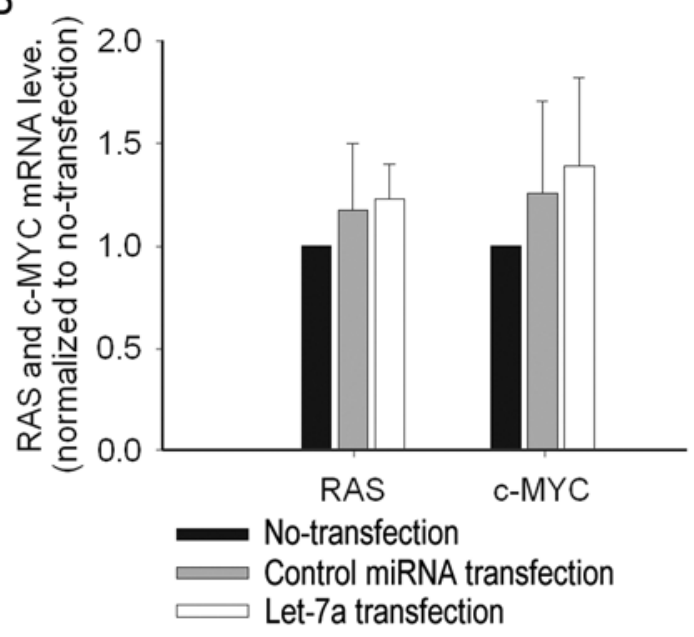

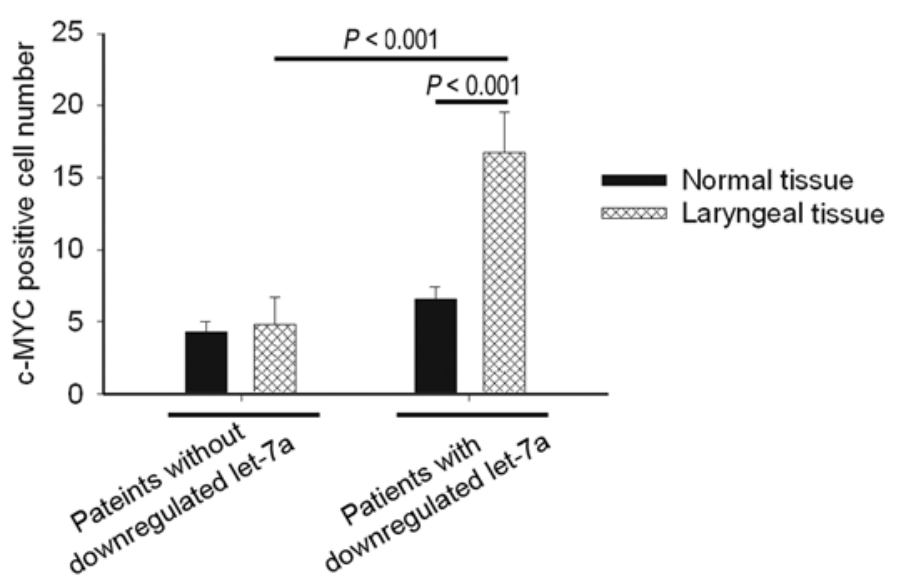

Figure 4. Let-7a miRNA inhibits the expression of RAS and c-MYC in laryngeal cancer. (A) Protein levels of RAS and c-MYC in Hep-2 cells at 48 h after transfection detected by Western blot analysis. One representative experiment of three independent experiments is shown. (B) mRNA levels of RAS and c-MYC evaluated by quantitative RT-PCR at $48 \mathrm{~h}$ after transfection (three independent experiments). (C) The number of RAS and c-MYC-positive cells in normal tissues $(n=48)$ and laryngeal cancer tissues with $(n=37)$ and without $(n=11)$ downregulated let-7a.

The let-7a miRNA inhibits proliferation, promotes apoptosis and increases DDP susceptibility of Hep-2 cells. Since let-7a was downregulated in both laryngeal cancer tissues and Hep-2 cells, we assessed whether let-7a could modulate the proliferation, apoptosis and chemotherapeutic drug sensitivity of Hep-2 cells. Firstly, we showed that let-7a mimics transfection could significantly increase the let-7a levels in Hep-2 cells, whereas control miRNA transfection displayed no obvious effect; when compared with cells without any transfection (Fig. 3A). Secondly, we found that the proliferation of Hep-2 cells was significantly inhibited, whereas the apoptosis of Hep-2 cells was markedly induced in let-7a-transfected cells compared with control miRNA-transfected cells and cells without any transfection (Fig. 3B, C and D). Moreover, overexpression of let-7a has a synergic effect with DDP on inhibition of Hep-2 cell proliferation and promotion of Hep-2 cell apoptosis (Fig. 3B, C and D).

RAS and c-MYC are target genes of let-7a in human laryngeal cancer. Since sequence analysis indicated that oncogenes RAS and c-MYC might be target genes of let-7a $(3,11)$, we investigated whether this hold true in laryngeal carcinoma. Firstly, we explored the direct effect of let-7a on RAS and c-MYC protein expression in Hep-2 cells. We found that RAS and c-MYC protein expression was significantly inhibited in Hep-2 cells transfected with let-7a compared with cells without any transfection. Nevertheless, no obvious effect was observed for control miRNA (Fig. 4A). In contrast to findings of protein levels, no apparent change in the mRNA levels of RAS and c-MYC was found after overexpression of let-7a in Hep-2 cells (Fig. 4B), indicating that let-7a may suppress RAS and c-MYC expression at the translation level. Secondly, we studied the RAS and c-MYC protein expression in laryngeal cancer and normal tissues by the means of immunohistochemistry. Since there were 11 patients who did not show downregulated expression of let-7a in tumor tissues as compared with paired adjacent normal tissues, we divided patients into two subtypes: patients with and without downregulated let-7a. We found that compared with normal tissues; there was a significantly stronger staining of RAS and c-MYC in cancer tissues with downregulated let-7a expression, than in cancer tissues without downregulated let-7a (Fig. 4C). 


\section{Discussion}

Increasing evidence has shown that miRNAs may be directly implicated in the carcinogenic process (18). More than 50\% of miRNAs are located in cancer-associated genomic regions or in fragile sites (19). A number of miRNAs promote whereas some other miRNAs inhibit cell proliferation and survival. These two classes of miRNAs may play an important role in cancer development as novel oncogenes and tumor suppressors, respectively $(5,6,18)$. The relationship between miRNA and tumor is attracting more and more interest. Previous studies have suggested that let-7 miRNA may be a tumor suppressor, which is poorly expressed in lung, colon and breast cancer $(10,11,20)$. Downregulated let-7 miRNA expression has been reported to have a prognostic impact on the survival of surgically treated lung cancer patients (10). Laryngeal cancer is a common malignant tumor of head and neck and 90-95\% of cancer tissues demonstrate squamous cell carcinoma. In this study, we found that compared with adjacent normal tissues, the let-7a expression was significantly reduced in laryngeal squamous cancer tissues. Moreover, a similar reduction was found in human laryngeal cancer cell line Hep-2 in comparison with normal bronchial epithelial cell line BEAS-2B, suggesting that let-7a downregulation is involved in laryngeal squamous carcinoma. Furthermore, we found a further decreased let-7a expression in non-differentiated tumors compared with moderately and well differentiated tumors, indicating a potential role of let- $7 \mathrm{a}$ in the cell differentiation of laryngeal squamous carcinoma. Nevertheless, it should be noted that in our study, there were still 11 patients $(23 \%)$ demonstrating unchanged or even slightly elevated expression levels of let-7a in tumor tissues compared with paired adjacent normal tissues, indicating that let-7a may exert different impacts on different individuals and let-7a may not take part in the pathogenesis of all laryngeal carcinoma. The reasons for the discrepancy in let-7a expression levels between different individuals with laryngeal squamous carcinoma need further study.

Since let-7 is essential in timing cell fate, we next explored whether let-7a play a role in the proliferation and apoptosis of laryngeal cancer cells. In this study, we did find that let-7a could suppress the growth and induce the apoptosis of laryngeal cancer cell, supporting the role of let-7a in laryngeal carcinoma development. Moreover, we found that let-7a could increase the sensitivity of Hep-2 cells to chemotherapeutic drug DDP, indicating that let-7a may be an important factor influencing the sensitivity of cells to chemotherapeutic drugs. Our findings are consistent with those in lung and colon cancer cells $(10,11)$; however, in contrast to the findings in human hepatocellular carcinoma HepG2 cells, in which let-7a suppressed the drug-induced apoptosis by targeting caspase-3 mRNA (21). These discrepancies indicate that let-7a may display different functions through interfering distinct genes in different cell types.

Sequence analysis suggests that oncogenes RAS and cMYC may be the targets of let-7a $(3,11)$. RAS proteins are membrane-associated GTPase, which are signaling proteins that regulate cellular growth and differentiation (22). MYC is an evolutionarily conserved nuclear protein also involved in the control of cell proliferation and differentiation (23).
Increased RAS and c-MYC expression has been demonstrated in laryngeal squamous carcinoma, especially in Asian patients, by some previous publications $(14,15,24-26)$. In the present study, employing let-7a transfection, we confirmed that let-7a can inhibit RAS and c-MYC protein expression at the translation level in Hep-2 cells. Moreover, we demonstrated that RAS and c-MYC protein expression was only increased in laryngeal cancer with decreased let-7a expression in Chinese patients, highlighting an inverse correlation between RAS and c-MYC protein and let-7a miRNA expression in laryngeal cancer tissues. These results suggest that elevated RAS and c-MYC protein expression may result from, at least in part, the loss of let-7a miRNA expression in laryngeal carcinoma. On the other hand, given the involvement of RAS and c-MYC in cell proliferation, the effect of let-7a on cell proliferation and apoptosis may be partially derived from its effect on RAS and c-MYC protein expression regulation. However, the factors contributing to the abnormal let-7a expression in laryngeal carcinoma remain to be defined.

In conclusion, in this study, for the first time, the let-7a miRNA expression is found to be downregulated in human laryngeal squamous carcinoma. Let-7a may inhibit the growth and induced the apoptosis of laryngeal cancer cell possibly through downregulating the protein expression of oncogenes RAS and c-MYC. Let-7a increases the susceptibility of laryngeal cancer cells to chemotherapeutic drugs and may be a potential therapeutic target for laryngeal carcinoma.

\section{Acknowledgements}

This study was supported by program for New Century Excellent Talents in University from State Education Ministry (NCET-07-0326 to Dr Zheng Liu).

\section{References}

1. Zamore PD and Haley B: Ribo-gnome: the big world of small RNAs. Science 309: 1519-1524, 2005.

2. Chang TC, Wentzel EA, Kent OA, et al: Transactivation of miR-34a by p53 broadly influences gene expression and promotes apoptosis. Mol Cell 26: 745-752, 2007.

3. Johnson SM, Grosshans H, Shingara J, et al: RAS is regulated by the let-7 microRNA family. Cell 120: 635-647, 2005.

4. Si ML, Zhu S, Wu H, Lu Z, Wu F and Mo YY: miR-21-mediated tumor growth. Oncogene 26: 2799-2803, 2007.

5. Lee YS and Dutta A: MicroRNAs: small but potent oncogenes or tumor suppressors. Curr Opin Investig Drugs 7: 560-564, 2006.

6. McManus MT: MicroRNAs and cancer. Semin Cancer Biol 13: 253-258, 2003.

7. Reinhart BJ, Slack FJ, Basson M, et al: The 21-nucleotide let-7 RNA regulates developmental timing in Caenorhabditis elegans. Nature 403: 901-906, 2000.

8. Pasquinelli AE, Reinhart BJ, Slack F, et al: Conservation of the sequence and temporal expression of let-7 heterochronic regulatory RNA. Nature 408: 86-89, 2000.

9. Lee YS, Kim HK, Chung S, Kim KS and Dutta A: Depletion of human micro-RNA miR-125b reveals that it is critical for the proliferation of differentiated cells but not for the down-regulation of putative targets during differentiation. J Biol Chem 280: 16635-16641, 2005.

10. Takamizawa J, Konishi H, Yanagisawa K, et al: Reduced expression of the let-7 microRNAs in human lung cancers in association with shortened postoperative survival. Cancer Res 64: 3753-3756, 2004

11. Akao Y, Nakagawa $Y$ and Naoe T: let-7 microRNA functions as a potential growth suppressor in human colon cancer cells. Biol Pharm Bull 29: 903-906, 2006. 
12. Sampson VB, Rong NH, Han J, et al: MicroRNA let-7a downregulates MYC and reverts MYC-induced growth in Burkitt lymphoma cells. Cancer Res 67: 9762-9770, 2007.

13. Crowe DL, Hacia JG, Hsieh CL, Sinha UK and Rice H: Molecular pathology of head and neck cancer. Histol Histopathol 17: 909-914, 2002

14. Ruiz-Godoy RL, Garcia-Cuellar CM, Herrera Gonzalez NE, et al: Mutational analysis of K-ras and Ras protein expression in larynx squamous cell carcinoma. J Exp Clin Cancer Res 25: $73-78,2006$

15. Krecicki T, Fraczek M, Jelen M, Zatonski T, Szkudlarek T and Dus D: Expression of c-myc oncoprotein in laryngeal squamous cell carcinoma. Acta Oto-Laryngologica 124: 634-637, 2004

16. Thomson JM, Parker J, Perou CM and Hammond SM: A custom microarray platform for analysis of microRNA gene expression. Nat Methods 1: 47-53, 2004.

17. Liu Z, Lu X, Zhang XH, et al: Clara cell $10-\mathrm{kDa}$ protein expression in chronic rhinosinusitis and its cytokine-driven regulation in sinonasal mucosa. Allergy 64: 149-157, 2009.

18. Cowland JB, Hother C and Gronbaek K: MicroRNAs and cancer. APMIS 115: 1090-1106, 2007.

19. Calin GA, Sevignani C, Dumitru CD, et al: Human microRNA genes are frequently located at fragile sites and genomic regions involved in cancers. Proc Natl Acad Sci USA 101: 2999-3004, 2004
20. Iorio MV, Ferracin M, Liu CG, et al: MicroRNA gene expression deregulation in human breast cancer. Cancer Res 65: 7065-7070, 2005.

21. Tsang WP and Kwok TT: Let-7a microRNA suppresses therapeutics-induced cancer cell death by targeting caspase- 3 . Apoptosis 13: 1215-1222, 2008.

22. Esquela-Kerscher A and Slack FJ: Oncomirs - microRNAs with a role in cancer. Nat Rev 6: 259-269, 2006.

23. Luscher B and Eisenman RN: New light on Myc and Myb. Part II. Myb. Genes Dev 4: 2235-2241, 1990.

24. Rizos E, Sourvinos G, Arvanitis DA, Velegrakis G and Spandidos DA: Low incidence of $\mathrm{H}-$, $\mathrm{K}$ - and $\mathrm{N}$-ras oncogene mutations in cytological specimens of laryngeal tumors. Oral Oncol 35: 561-563, 1999.

25. Saranath D, Chang SE, Bhoite LT, et al: High frequency mutation in codons 12 and 61 of H-ras oncogene in chewing tobacco-related human oral carcinoma in India. Br J Cancer 63 : 573-578, 1991 .

26. Kuo MY, Jeng JH, Chiang CP and Hahn LJ: Mutations of Ki-ras oncogene codon 12 in betel quid chewing-related human oral squamous cell carcinoma in Taiwan. J Oral Pathol Med 23: 70-74, 1994. 